\title{
Contact parameter analysis and calibration in discrete element simulation of rice straw
}

\author{
Honglei Jia ${ }^{1}$, Jiayu Deng ${ }^{1,2,3}$, Yanling Deng ${ }^{2}$, Tianyou Chen ${ }^{1}$, Gang Wang ${ }^{1 *}$, \\ Zaijin Sun ${ }^{2}$, Hui Guo ${ }^{1}$ \\ (1. College of Biological and Agricultural Engineering, Jilin University, Changchun 130022, China; \\ 2. Technical Center for Soil, Agricultural and Rural Ecology and Environment, Ministry of Ecology and Environment of \\ the People's Republic China, Beijing 100012, China; \\ 3. College of Mechanical Engineering, Harbin Institute of Petroleum, Harbin 150028, China)
}

\begin{abstract}
Discrete element method was used to study and analyze the interaction between rice straws and between rice straw and agricultural machinery parts, thereby providing a scientific basis for post-harvest paddy field processing. Calibrations of rice straw-rice straw, rice straw-agricultural machinery part contact parameters (collision recovery coefficient, static friction coefficient and rolling friction coefficient) constitute an important prerequisite for the discrete element research process. In this study, the collision recovery coefficients of rice straw-steel and rice straw-rice straw were 0.230 and 0.357 , respectively, which were calibrated by the collision method. The static friction coefficient and rolling friction coefficient of rice straw-steel were 0.363 and 0.208 respectively, which were calibrated by the inclined plate method and the slope method. The static friction coefficient and rolling friction coefficient of rice straw-rice straw were 0.44 and 0.07 , respectively, which were calibrated by the split cylinder method. The paired $t$-test showed insignificant differences between calibration parameter simulation results and the physical test values $(p>0.05)$. Taking the angle of repose that reflecting rice straw flow and friction characteristics as the evaluation index, the verification tests of the above calibration values indicated that the simulated angle of repose has no significant difference from the physical test value $(p>0.05)$. The side plate lifting test on rice straw of different lengths showed no significant difference between the simulated angle of repose and the physical test value $(p>0.05)$. This study can provide a basis for contact parameters choice in discrete element simulation analysis with rice straw-rice straw and rice straw-agricultural machinery parts as the research object. The calibration method can provide a reference for the contact parameter calibration of other crop straws.
\end{abstract}

Keywords: rice straw, discrete element simulation, contact parameter, parameter calibration, angle of repose DOI: $10.25165 /$ j.ijabe.20211404.6435

Citation: Jia H L, Deng J Y, Deng Y L, Chen T Y, Wang G, Sun Z J, et al. Contact parameter analysis and calibration in discrete element simulation of rice straw. Int J Agric \& Biol Eng, 2021; 14(4): 72-81.

\section{Introduction}

In post-harvest rice processing, rice straw picking, bundling as well as returning are important links in modern agriculture ${ }^{[1-3]}$. Studying the interaction of rice straw-rice straw and rice straw-agricultural machinery parts is a basic scientific issue in the development and improvement of rice post-harvest processing equipment $^{[4-7]}$. In recent years, the simulation analysis technology

Received date: 2021-01-11 Accepted date: 2021-05-14

Biographies: Honglei Jia, PhD, Professor, research interest: bionic intelligent agricultural machinery and conservation tillage technology, Email: jiahl@ vip.163.com; Jiayu Deng, PhD candidate, Lecturer, research interest: intelligent agricultural machinery and its key technology, Email: dengjychn@163.com; Yanling Deng, PhD, Research Associate, research interest: ecological protection of agricultural land, Email: 419960321@qq.com; Tianyou Chen, PhD candidate, research interest: straw resource utilization, Email: chentianyou93@ 163.com; Zaijin Sun, PhD, Associate Researcher, research interest: risk management and control of soil pollution in agricultural land, Email: szj0007@163.com; Hui Guo, PhD, Engineer, research interest: Agricultural machinery and its mechanism of action, Email: guohuipp0322@163.com.

*Corresponding author: Gang Wang, $\mathrm{PhD}$, Associate Professor, research interest: intelligent agricultural machinery and its key technology. College of Biological and Agricultural Engineering, Jilin University, Changchun 130022, China. Tel: +86-13166888540, Email: wangganggoodman@163.com. of discrete element method (DEM) receives great attention in the design and improvement of agricultural machinery parts. DEM can accurately analyze the movement law of agricultural materials ${ }^{[8,9]}$, provide a microscopic visual analysis of rice straw particles, dynamic meso-contact behavior between rice straw and agricultural machinery parts, thereby providing technical parameters for trial production of physical prototypes and avoiding structural problems before trial production. However, in discrete element simulation, contact parameters (collision recovery coefficient, static friction coefficient, and rolling friction coefficient) of rice straw-rice straw and rice straw-agricultural machinery parts cannot be directly determined by physical tests. The rationality of these parameters directly affects the accuracy of the simulation results ${ }^{[10,11]}$. At this stage, research on contact parameters of rice straw-rice straw and rice straw-agricultural machinery parts is still rarely reported. Domestic and foreign scholars have carried out the following research on the selection and calibration of straw contact parameters.

Fang $^{[12]}$ determined the wheat straw contact parameters with reference to Lenaerts et $\mathrm{al}^{[13]}$. DEM simulation was used to study the interaction mechanism of wheat straw-soil-rotary tiller. By analyzing rotary tiller force in three directions, it was concluded that increased direct straw burial in operations could reduce energy 
consumption in straw burial via repeated rotary tillage. Zhang ${ }^{[14]}$ determine the contact parameters of rice straw-soil-rotary tiller roller by empirical values, and used DEM simulation to analyze the combined knife roller operation process in high straw returning. Liu et al. ${ }^{[15]}$ selected wheat straw-steel contact parameters to establish a flexible wheat straw model using DEM and evaluate the impact of the calibrated bonding parameters on the model. The results indicated that flexible wheat straw could accurately reflect wheat straw bending property. Zhou et al. ${ }^{[16]}$ cited contact parameter value in literature ${ }^{[14]}$ to conduct simulation and experimental research on the spatial distribution effect after straw returning. Using the orthogonal method, Zhang et al. ${ }^{[17]}$ calibrated the contact parameters between maize stalks and between maize stalks and crusher hammer, finding a relative error of $8.127 \%$ between the simulated and experimental value of the radial accumulation angle. With the angle of repose as the basis, Wu et al. ${ }^{[18]}$ calibrated the discrete element parameters of tomato straw by orthogonal method to reveal the vertical spiral mixing mechanism of tomato straw.

In summary, at present, discrete element contact parameters of rice straw mostly adopt universal value or empirical value, and the large errors directly affect the accuracy of simulation analysis. To more accurately determine the contact parameters of rice straw-rice straw, rice straw-agricultural machinery parts, this study adopted virtual calibration method ${ }^{[19]}$, provided measured macro index values based on physical test, set simulation model within the measured macro index value range, and then conducted physical test and simulation test simultaneously based on specific contact parameter values of rice straw-rice straw and rice straw-agricultural machinery parts. In case of difference between the two, the target contact parameter value will be corrected and adjusted to finally determine calibrated contact parameter ${ }^{[20]}$. That is, first, determining the contact parameter level value in the simulation test plan according to the value range of some physical test-derived contact parameters. Then, use the measured index value as the simulation test target to calculate the rice straw-steel contact parameter and rice straw-rice straw collision recovery coefficient, perform significance analysis on the simulated value and the physical test value under the calibrated values. Secondly, based on Central Composite Design (CCD), the value range of the rice straw-rice straw static friction coefficient and rolling friction coefficient derived from the pre-simulation test was used as the factor range of the simulation test. The angle of repose reflecting rice straw flow and friction characteristics is taken as the evaluation index for the simulation test. The simulation results were subject to variance analysis, and the angle of repose measured by the split cylinder method was used as the target value for optimization of the simulation regression model to get the optimized contact parameter calibration value. Finally, the verify accuracy of the above calibrated contact parameters as well as the feasibility of the calibration method; used side plate lifting test to verify universal applicability of the calibration value and the calibration method to rice straw of different lengths.

\section{Materials and methods}

\subsection{Establishment of rice straw model}

The study found that the length of harvested rice straw should be no more than $100 \mathrm{~mm}^{[21]}$, and that of rice straw for other purposes should generally be no more than $30 \mathrm{~mm}^{[22]}$. The rice straw sample in this study is natural air-dried rice straw (Miaodao No. 2) taken from the Agricultural Experimental Base of Jilin
University in May $2020\left(125^{\circ} 15^{\prime} \mathrm{E}, 43^{\circ} 57 \mathrm{~N}\right)$. A randomized complete block design with six replicates was established in the field, and then five one-square-meter matrices were selected randomly. Considering simulation efficiency and rice straw consistency, the rice straw with an internode length of $10 \mathrm{~mm}$ was taken as the research object ${ }^{[23]}$. Fifty internode rice straws were randomly intercepted in each matrice ( 5 matrices $\times 6$ replicates, 30 in total), all rice straws were mixed evenly and sampled by the quartering method before the test. The moisture content of rice straw measured by XY-102MW halogen moisture analyzer was $10.83 \%$. Bonding effect between rice straws can be ignored, so Hertz-Mindlin (no slip) contact model in the discrete element simulation software EDEM was selected ${ }^{[24]}$ to establish the rice straw model as shown in Figure 1. The rice straw appearance observed under the ZEISS stereo microscope is shown in Figure 1a. The rice straw was approximately cylindrical with outer striped protrusions. The current straw models mostly adopt multi-balls that come with the software to form straw strips ${ }^{[13,14,16,18,23]}$, which were quite different from the actual straw in shape. In this study, the rice straw Pro/E three-dimensional model was imported into EDEM software. As shown in Figure $1 \mathrm{~b}$, the $10 \mathrm{~mm}$ long rice straw model filled with randomly generated small balls was established with model contour as the filling boundary. According to the average segment diameter of the sample, the model diameter was determined to be $5.5 \mathrm{~mm}$.

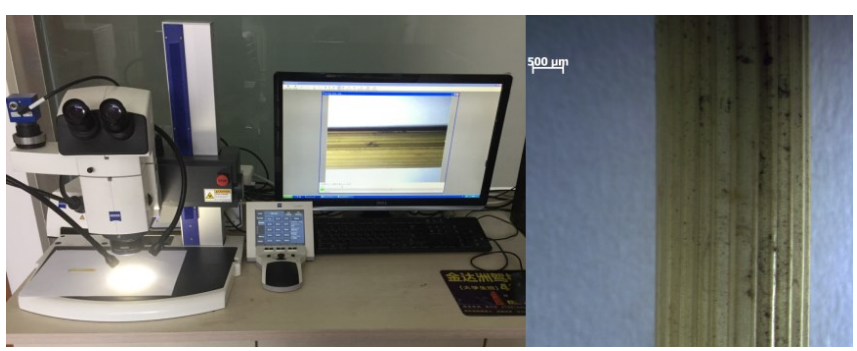

a. Observation test on the outer surface of rice straw
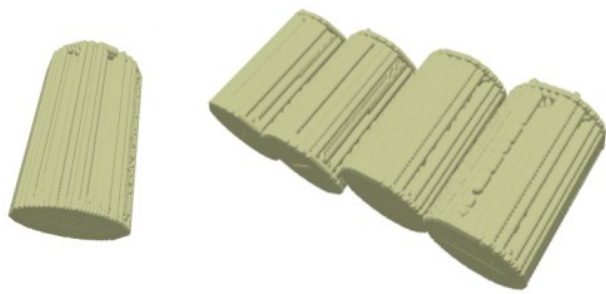

b. Single rice straw and rice straw row model

Figure 1 Establishment of discrete element model of rice straw

\subsection{Determination of intrinsic parameters in discrete element simulation}

The material property parameters required in EDEM simulation include intrinsic parameters and contact parameters. The test process involved the contact between rice straw and rice straw, and between rice straw and collision surface. The contact material was Q235 steel commonly used in agricultural machinery ${ }^{[25]}$.

Intrinsic parameters mean characteristics of the material itself, which are usually relatively fixed. Rice straw density is measured by the liquid displacement method ${ }^{[26]}$, and intrinsic parameters required for the simulation herein are determined as listed in Table 1 through experiments and literature consultation ${ }^{[14,15,27,28]}$. Material contact parameters are related to objects in contact. The contact parameters calibrated herein include collision recovery coefficient, static friction coefficient and rolling friction coefficient of rice straw-steel and rice straw-rice straw. 
Table 1 Intrinsic parameters of materials

\begin{tabular}{lccc}
\hline \multirow{2}{*}{ Material } & \multicolumn{3}{c}{ Intrinsic parameter } \\
\cline { 2 - 4 } & Poisson's ratio & Density $/ \mathrm{kg} \cdot \mathrm{m}^{-3}$ & Shear modulus $/ \mathrm{Pa}$ \\
\hline Rice straw & $0.4^{[14,15]}$ & $196^{\mathrm{a}}$ & $1 \times 10^{6[14,27]}$ \\
Steel & $0.3^{[14,15,28]}$ & $7865^{[14,28]}$ & $7.9 \times 10^{10[14,28]}$ \\
\hline
\end{tabular}

Note: The values marked with the letter ${ }^{a}$ in the table are the values measured by experiments.

\subsection{Calibration test of rice straw-steel contact parameters}

\subsubsection{Rice straw-steel collision test}

Collision recovery coefficient is a parameter that reflects the ability to return to its original shape after the collision between particles or between particles and objects. It can be expressed as the ratio of the separation speed of the two objects after the collision to the approaching speed before the collision ${ }^{[29,30]}$.

$$
e=\frac{v_{2}-v_{1}}{v_{1}^{0}-v_{2}^{0}}
$$

where, $e$ is the collision recovery coefficient; $v_{1}^{0}$ and $v_{2}^{0}$ are the speeds before the collision of object 1 and object 2 , respectively, $\mathrm{m} / \mathrm{s} ; v_{1}$ and $v_{2}$ are the speeds after the collision of object 1 and object 2 , respectively, $\mathrm{m} / \mathrm{s}$.

Material collision recovery coefficient is commonly determined by free fall collision method or inclined plate collision method, which requires normal material rebound after falling collision $^{[17,23,31,32]}$. However, rice straw anisotropy makes accurate rebound difficult after a collision. Therefore, based on the law of conservation of kinetic energy, this study adopted a simple pendulum test similar to the Newtonian pendulum test in principle to determine the rice straw-steel collision recovery coefficient $e_{s}$. The test device is shown in Figure 2. Wang ${ }^{[33]}$ found that steel plate thickness has an insignificant effect on $e$, so the test used a steel plate of $100 \mathrm{~mm} \times 100 \mathrm{~mm} \times 8 \mathrm{~mm}$. Before the test, super glue was used to fix the rice straw on one end of a nylon wire perpendicular to the rice straw axis with a diameter of 0.25 $\mathrm{mm}$, while the other end was fixed on a height-adjustable support

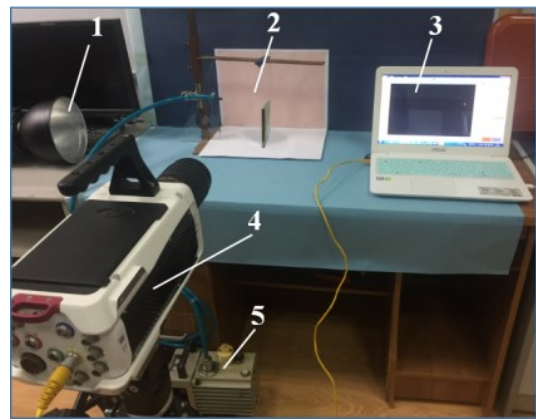

a. Test device

1. Fill light 2. Test area 3. PCC2.6 software 4. High-speed camera 5. Vacuum pump 6. Support 7. Suction nozzle 8. Rice straw 9. Graph paper 10. Steel plate 11. Nylon wire

Figure 2 Rice straw-steel collision test device

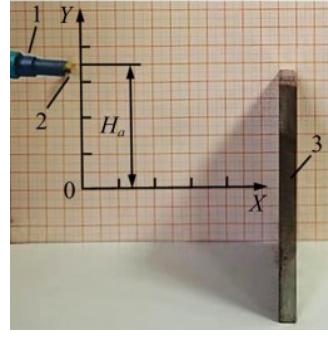

Initial release

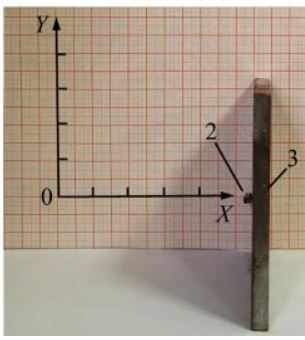

First collision cantilever. The nylon wire has a negligible energy dissipation effect on the system ${ }^{[33]}$. The collision process photo collected by a high-speed camera (Phantom v711, Vision Research, USA) is shown in Figure 3a. The vacuum pump suction nozzle adsorbs and maintains the rice straw at the release height, while the rice straw axis is kept parallel to the horizontal plane and the steel plate plane. After turning off the vacuum pump, the rice straw was released without initial velocity to collide with the steel plate for the first time, which then rebounded to the highest point. Equation (2) of $e_{s}$ can be derived from Equation (1), and $e_{s}$ value can be calculated based on value reading on millimeter graph paper.

$$
e_{s}=-\frac{v_{i}^{n}}{v_{j}}=\frac{\sqrt{2 g h_{a}}}{\sqrt{2 g H_{a}}}=\sqrt{\frac{h_{a}}{H_{a}}}
$$

where, $e_{s}$ is the rice straw-steel collision recovery coefficient; $v_{i}^{n}$ and $v_{j}$ are the normal rebound velocity and incident velocity of the rice straw, m/s; $H_{a}$ and $h_{a}$ are the rice straw release height and rebound height, respectively, mm; $g$ is the acceleration of gravity, $\mathrm{m} / \mathrm{s}^{2}$.

The collision simulation test is shown in Figure $3 \mathrm{~b}$. In the equivalent collision model, a pipeline centerline plane is a vertical plane, the pipeline section is a rectangle of $14 \mathrm{~mm} \times 8 \mathrm{~mm}$, and the closed right end of the pipeline is the collision plane. Wang ${ }^{[33]}$ found that the intrinsic parameters of the pipeline had insignificant effects on the collision process, according to the steel setting. The rice straw-steel friction coefficient was set to $0^{[19]}$. According to EDEM user manual, the simulation time steps herein were all set to $20 \%$ of the rayleigh time step, and the grid size was 2.5 times of the minimum particle radius. The rice straw is confined in a $1 / 4$ circular pipeline with a radius of $200 \mathrm{~mm}$, and a rice straw model was generated at the vertical height $H_{a}$ from the lowest point of the pipeline. The rice straw was released without initial velocity, which rebounded to the highest point after colliding with the right end face of the pipeline. $h_{a}$ value was calculated after software post-processing.

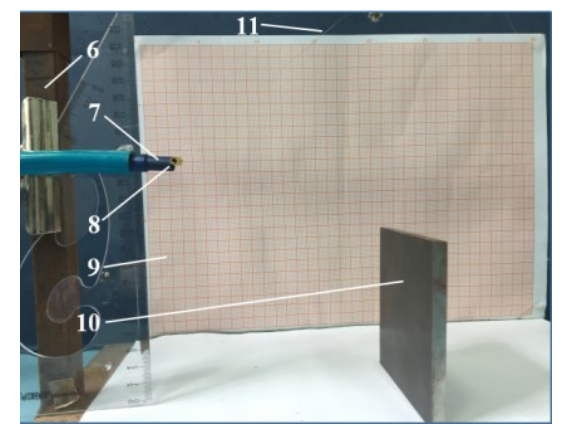

b. Enlarged test area

test device

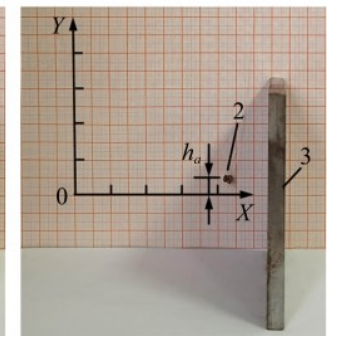

Highest point of rebound

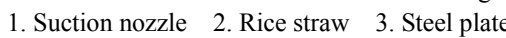

Note: Each scale increment on $X$ - and $Y$-axes is $20 \mathrm{~mm}$, the same as below.

a. Physical test 


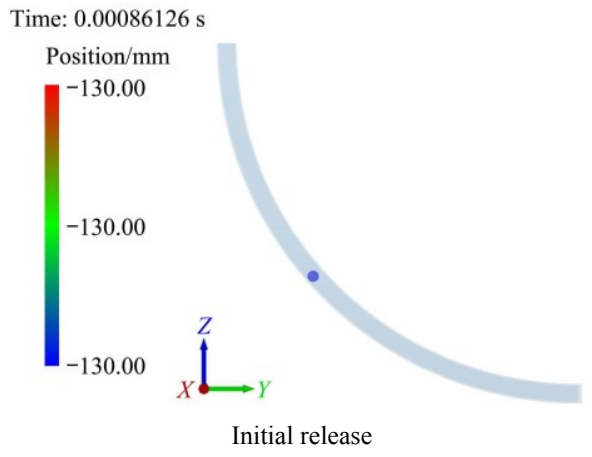

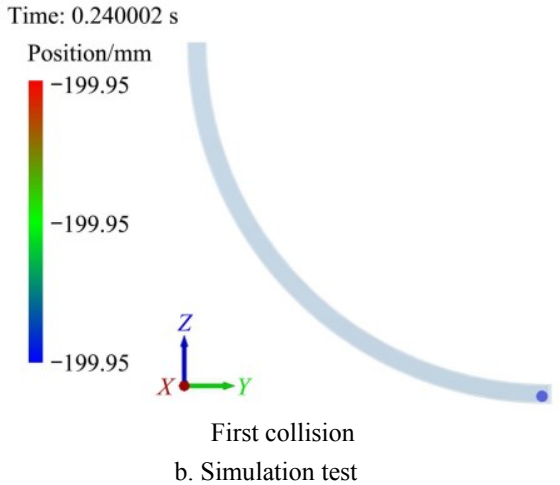

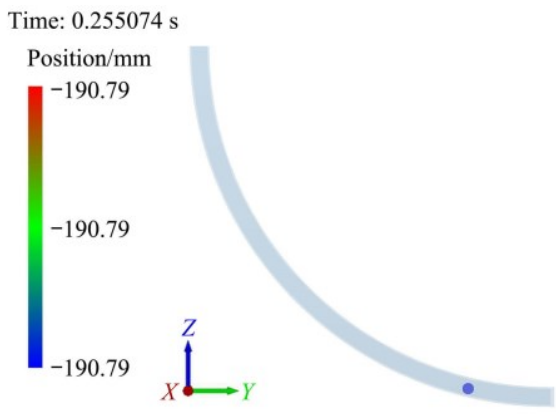

Highest point of rebound

Figure 3 Comparison of rice straw-steel collision test

\subsubsection{Rice straw-steel inclined plate test}

The rice straw-steel static friction physical test by the inclined plate method is shown in Figure 4a. The steel plate is fixed in the groove of the self-made inclined plate instrument. To prevent rice straw rolling, the four sections of rice straw are bonded into rice straw row and placed on the steel plate. The inclined plate is slowly raised at a constant speed, the digital display goniometer displays the value of the inclination angle $\alpha_{1}$ in real-time, and the high-speed camera captures the instant value $\alpha_{1}$ when the rice straw row slide on the inclined plate. The tangent value is the rice straw-steel static friction coefficient, which is expressed as ${ }^{[23,34]}$ :

$$
\mu_{1}=\tan \alpha_{1}
$$

where, $\mu_{1}$ is the rice straw-steel static friction coefficient; $\alpha_{1}$ is the inclination angle of the inclined plate, $\left(^{\circ}\right)$.

The inclined plate simulation test is shown in Figure $4 \mathrm{~b} . e_{s}$ was set to the calibration value in Section 2.3.1, and the rice straw-steel rolling friction coefficient was set to 0 . The rice straw row shown in Figure 1d is generated on the surface of the steel plate model. The steel plate rotated counterclockwise around the $Z$-axis at a speed of $10^{\circ} / \mathrm{s}^{[19]}$, and the value $\alpha_{1}$ was recorded when the rice straw row had a sliding trend.

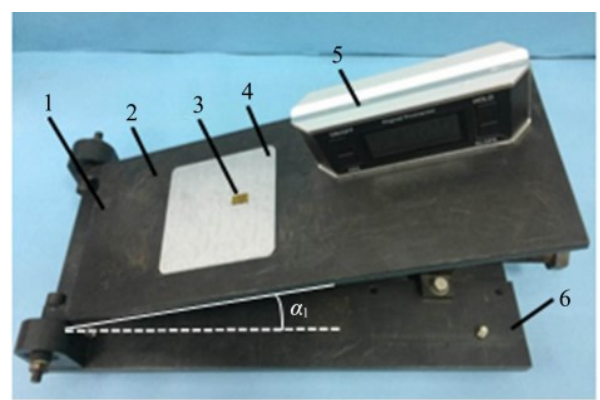

1. Self-made inclined plate instrument 2. Inclined plate 3. Rice straw row 4. Steel plate 5. Digital display goniometer 6. Plate

$$
\text { a. Physical test }
$$

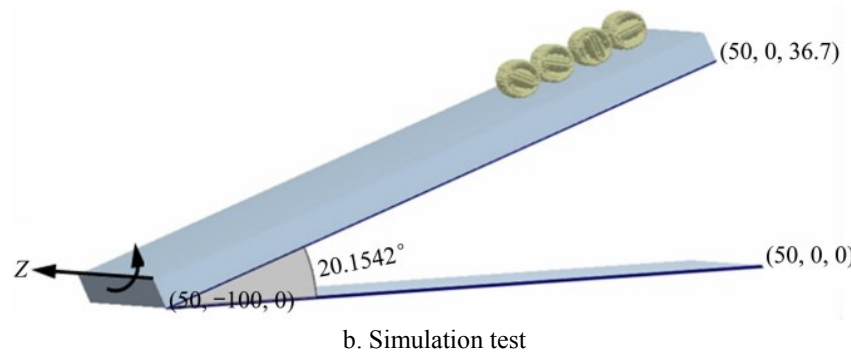

Figure 4 Rice straw-steel static friction test

\subsubsection{Rice straw-steel slope test}

The rice straw-steel rolling friction physical test by slope method is shown in Figure 5a. The rice straw is released from the inclined plate at a distance $s_{0}$ from the intersection line without initial velocity, which, driven by resistance, rests on the plate with distance $s$ from the intersection. Equation (4) can be derived from the law of conservation of energy, and Equation (5) of the rice straw-steel rolling friction coefficient $\mu_{1}^{\prime}$ can be derived ${ }^{[35,36]}$. In the test, to prevent rice straw bouncing due to excessively inclined angle $\beta$, or insufficient rolling due to too small $\beta, s_{0}$ is selected as $100 \mathrm{~mm}$ and $\beta$ is selected as $30^{\circ}$ through a trial test. The rolling distance $s$ is measured and substituted into Equation (5) to get $\mu_{1}^{\prime}$.

$$
\mu_{1}^{\prime}=\frac{s_{0} \cdot \sin \beta}{s_{0} \cdot \cos \beta+s}
$$$$
m g \sin \beta \cdot s_{0}=\mu_{1}^{\prime} m g \operatorname{con} \beta \cdot s_{0}+\mu_{1}^{\prime} m g \cdot s
$$

where, $\mu_{1}^{\prime}$ is the rice straw-steel rolling friction coefficient; $s_{0}$ is the distance from the initial rice straw position to the intersection line, $\mathrm{mm} ; s$ is the rice straw rolling distance on the plate, $\mathrm{mm} ; \beta$ is the slope inclination angle, $\left(^{\circ}\right)$.

The slope simulation test is shown in Figure 5b. $e_{s}$ was set as the calibration value in Section 2.3.1, and $\mu_{1}$ was set as the calibration value in Section 2.3.2. The rice straw was released where $s_{0}$ was $100 \mathrm{~mm}$ without initial velocity. The rice straw rests on the plate after rolling. The legend in the figure shows the real-time rolling distance of the rice straw, and the $s$ value under static rice straw was recorded.

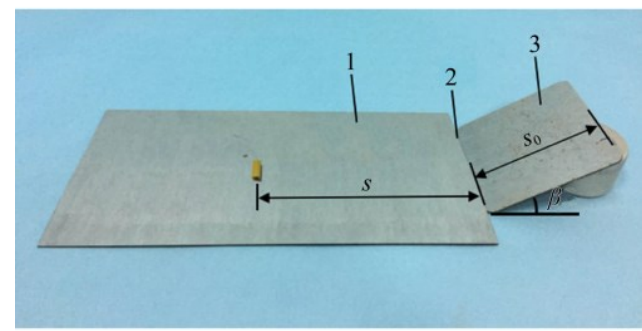

1. Plate 2. Intersection line 3. Inclined plate

Note: $s$ is the rice straw rolling distance on the plate, $\mathrm{mm} ; s_{0}$ is the distance from the initial rice straw position to the intersection line, $\mathrm{mm} ; \beta$ is the slope inclination angle, $\left(^{\circ}\right)$.

$$
\text { a. Physical test }
$$

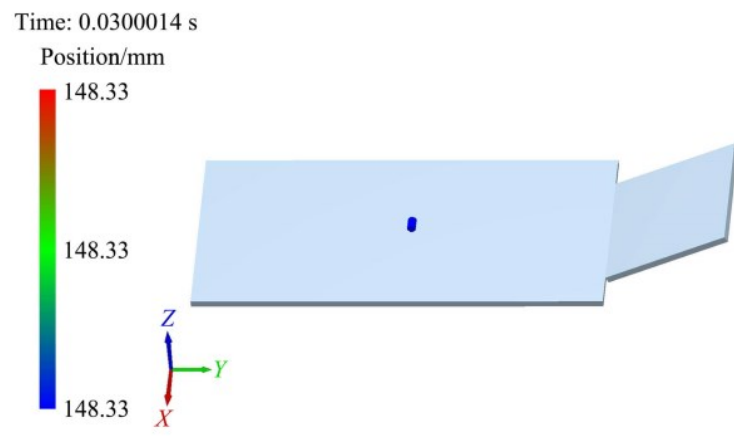

b. Simulation test

Figure 5 Rice straw-steel rolling friction test 


\subsection{Calibration test of rice straw-rice straw contact parameters}

2.4.1 Rice straw-rice straw collision test

Rice straw-rice straw collision recovery coefficient $e_{r}$ was determined by double pendulum test ${ }^{[33,37]}$ with test device the same as that in Figure 2. In the test area, two nylon wires of equal length were used as the cycloid, one end of which was suspended with two rice straws approximate in shape and size. The suspension angle, height and focus point are consistent. Figure 6a shows the physical test of rice straw collision under a high-speed camera. The rice straw with initial release height reached the highest point after the first collision. Equation (6) can be derived from Equation (1).

$$
e_{r}=\frac{v_{2}-v_{1}}{v_{1}^{0}}=\frac{\sqrt{2 g H_{c}}-\sqrt{2 g H_{b}}}{\sqrt{2 g H_{0}}}=\frac{\sqrt{H_{c}}-\sqrt{H_{b}}}{\sqrt{H_{0}}}
$$

where, $e_{r}$ is the rice straw-rice straw collision recovery coefficient; $H_{0}$ is the rice straw release height, $\mathrm{mm} ; H_{b}$ and $H_{c}$ are the heights of two rice straws after a collision, respectively, $\mathrm{mm}$.

As shown in Figure 6b, the collision simulation test adopts an equivalent collision mode ${ }^{[34]}$ with the basic settings the same as in section 2.3.1, in which the rice straw-steel contact parameter and the rice straw-rice straw friction coefficient are all set to 0 . The rice straw is confined in the semicircular pipeline with a radius of $200 \mathrm{~mm}$ as shown in Figure 6b. One rice straw model is generated at the lowest point of the pipeline and the other at the distance $H_{0}$ from the vertical height. The rice straw at $H_{0}$ is released without initial velocity, and the two rice straws reach the highest points $H_{b}$ and $H_{c}$ respectively after the first collision.

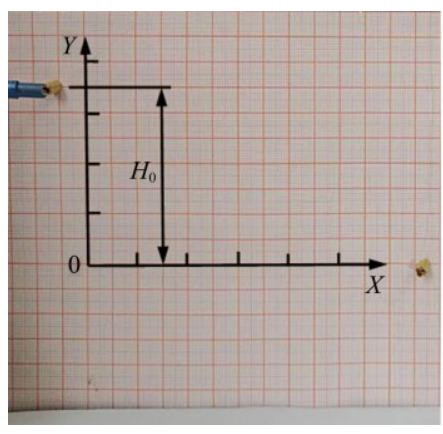

Initial release

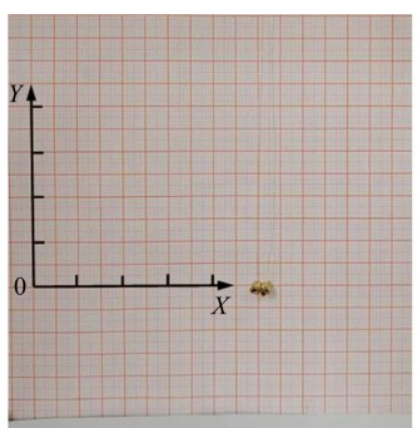

First collision a. Physical test

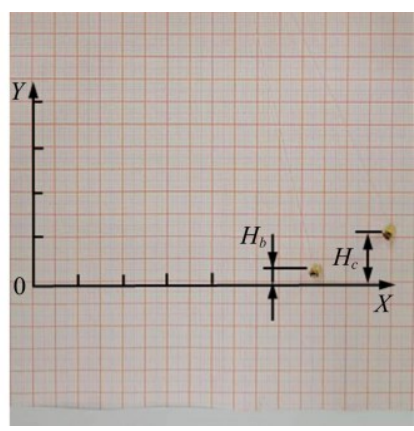

Highest point of collision

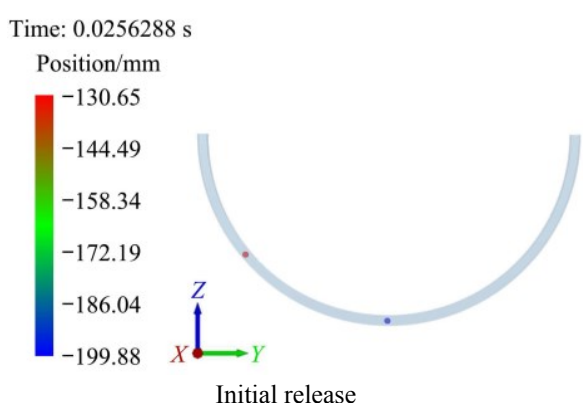

Time: $0.27 \mathrm{~s}$ Position $/ \mathrm{mm}$ Time: $0.480001 \mathrm{~s}$

Position $/ \mathrm{mm}$
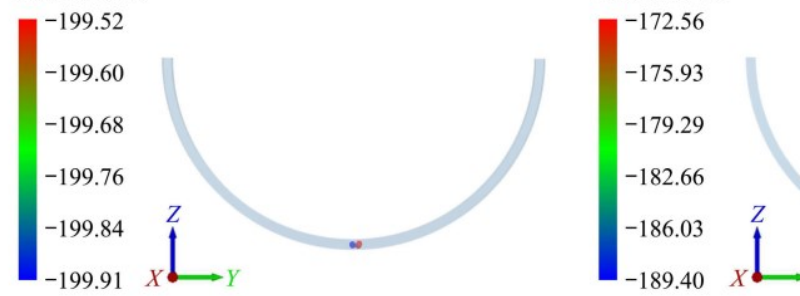

First collision

Highest point of rebound

Figure 6 Comparison of rice straw-rice straw collision test

\subsubsection{Rice straw-rice straw angle of repose test}

The natural angle of the repose of the material reflects its flow and friction characteristics ${ }^{[38]}$. Studies have shown a significant impact on the angle of repose from friction coefficient ${ }^{[24,39]}$. In this section, by the split cylinder method, the angle of repose test was used for calibration of rice straw-rice straw static friction coefficient $\mu_{2}$ and rolling friction coefficient $\mu_{2}^{\prime}{ }_{2}^{[39,40]}$.

The physical test is shown in Figure 7a. The steel hollow cylinder and the cylinder base have an inner diameter of $100 \mathrm{~mm}$, a wall thickness of $2 \mathrm{~mm}$, and a height of $65 \mathrm{~mm}$. Fill the cylinder with rice straw, and slowly lift the hollow cylinder at a uniform speed. Then, determining the mutually perpendicular generatrix of the stable cone formed by the rice straw pile in four directions, and take the average value of the inclination angle as the angle of repose.

The simulation test and settings are shown in Figures $7 \mathrm{~b}$ and 7c. The contact parameters $e_{s}, \mu_{1}, \mu_{1}^{\prime}$ and $e_{r}$ are set to the calibrated values above, $\mu_{2}$ and $\mu_{2}^{\prime}$ are set according to the experimental plan design. Rice straw particles are generated in the cylinder. After the particles are stable, the hollow cylinder is lifted vertically at a rate of $0.001 \mathrm{~m} / \mathrm{s}^{[38]}$. To avoid errors caused by direct measurement of rice straw pile, as shown in Figure 8, read the original image Figure $8 \mathrm{a}$ at the edge of the rice straw pile by Matlab, output the binary image Figure $8 b$ after sequential grayscale and binarization to generate the hole filling image Figure 8c. Using the least square method, the extracted boundary contour line is fitted to derive fitting image Figure $8 \mathrm{~d}$, and the slope of the fitting straight line is the tangent of the angle of repose. To facilitate the reading of the slope value, the horizontal and vertical rulers are set in Figure 8d.

\subsection{Data analysis}

In this study, Design-Expert 8.0.6.1 (Stat-Ease Inc., USA) was used for statistical analysis. A $t$-test was used to verify whether the simulation test results under calibrated contact parameters are significantly different from the physical test results in the collision test, inclined plate test, slope test, and side plate lifting test. $F$-test was used to test whether there is a statistical difference between the simulated angle of repose and the physical test value in the split cylinder method. The angle of repose in the test was read by Matlab 9.5.0 (MathWorks Inc., USA). OriginPro 2019b (OriginLab Inc., USA) was used to plot the impact of different release heights on the collision recovery coefficient. 


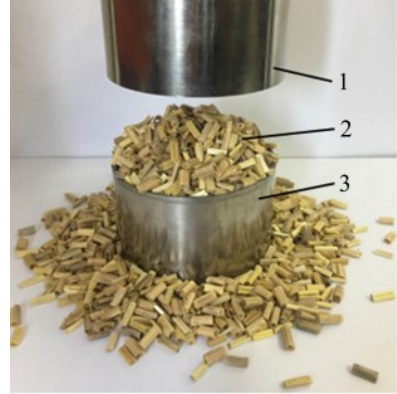

a. Physical test

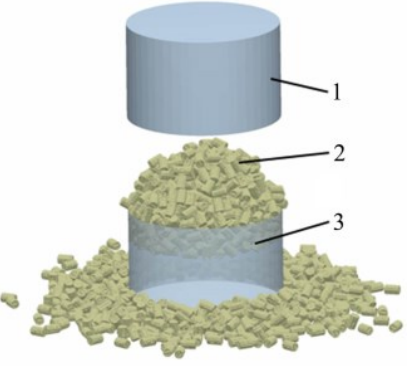

b. Simulation test

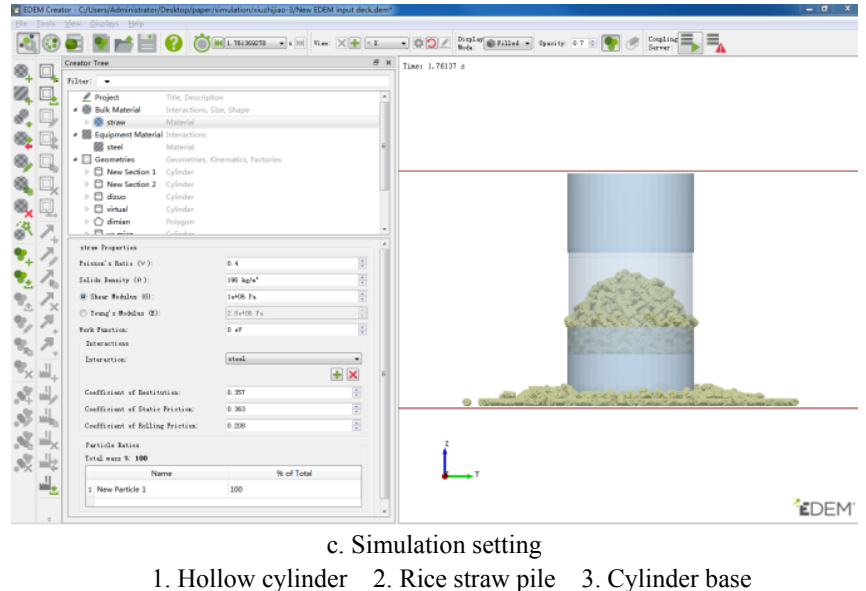

Figure 7 Angle of repose test by split cylinder method

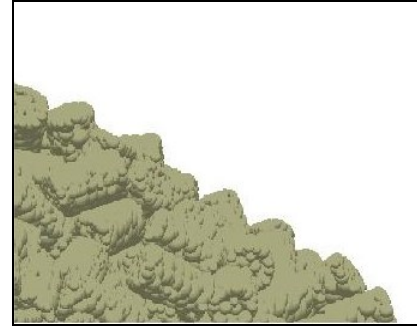

a. Original image

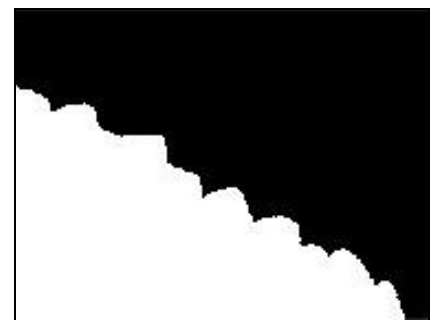

c. Hole filling image

Figure 8 Image processing by Matlab software

\section{Results and discussion}

\subsection{Analysis on calibration results of rice straw-steel contact} parameters

3.1.1 Rice straw-steel collision recovery coefficient

Three sets of rice straw-steel collision physical tests with $H_{a}$ at $40 \mathrm{~mm}, 70 \mathrm{~mm}$, and $100 \mathrm{~mm}$ are performed, with each test repeated 15 times to get average $h_{a}$ values of $5.33 \mathrm{~mm}, 9.17 \mathrm{~mm}, 12.96 \mathrm{~mm}$. The measured $e_{s}$ values were $0.365,0.362,0.360$, respectively. Lu et al. ${ }^{[29]}$ found that $H_{a}$ has no significant effect on $e$. Therefore, the simulation test is performed by setting $H_{a}=70 \mathrm{~mm}$ as the initial condition, then $h_{a}=9.17 \mathrm{~mm}$ as the simulation target value, and the measured $e_{s}$ under this condition has a range of 0.335-0.392. The increments in the various test levels were determined according to the research method of Lenaerts et al. ${ }^{[13]}$. $e_{s}$ level value and simulation results are listed in Table 2. With rice straw-steel collision recovery coefficient as the independent variable $x_{1}$ and the rebound height as the dependent variable $y_{a}$, the curve fitting equation for the simulation test is derived as:

$$
y_{a}=333.14 x_{1}^{2}-204.12 x_{1}+39.576
$$

Table 2 Simulation test results of rice straw-steel collision

\begin{tabular}{ccc}
\hline Group number & Recovery coefficient $x_{1}$ & First rebound height $y_{a} / \mathrm{mm}$ \\
\hline 1 & 0.335 & $8.63 \pm 0.04$ \\
2 & 0.349 & $8.82 \pm 0.04$ \\
3 & 0.363 & $9.38 \pm 0.05$ \\
4 & 0.377 & $10.06 \pm 0.06$ \\
5 & 0.392 & $10.71 \pm 0.05$ \\
\hline
\end{tabular}

The determination coefficient $R^{2}=0.993$. When $y_{a}=9.17$, then $x_{1}=0.357$. Set $e_{s}$ to 0.357 , and three sets of repeated simulation tests are performed with $H_{a}$ at $40 \mathrm{~mm}, 70 \mathrm{~mm}$, and $100 \mathrm{~mm}$ to get the average $h_{a}$ value of $5.25 \mathrm{~mm}, 8.94 \mathrm{~mm}$, and $12.56 \mathrm{~mm}$, respectively. The calculated $e_{s}$ values were $0.362,0.357$, and 0.354 , respectively. Paired $t$-test of $h_{a}$ simulated value and physical test value revealed an insignificant difference between the two ( $p>0.05)$, so $e_{s}$ calibration value was determined to be 0.357 .

Figure 9 shows the impact of different $H_{a}$ on $e_{s}$. $e_{s}$ has a smaller simulated value than the physical test value. The error between the two was because air resistance and operation error in the physical test lead to deviation of rice straw trajectory from the simulation test, which is consistent with the reason for errors in $e$ value of maize kernels in the study of Wang ${ }^{[33]}$. In addition, Wong et al. ${ }^{[37]}$ also showed that $e$ measurement is affected by air resistance. Rice straw outer surface has more evenly distributed strip-shaped protrusions in simulation than in physical test, which has line contact collision with the steel plate. The collision contact is larger compared to the physical test, with big energy loss, reduced rebound speed, so the simulated value is smaller than the physical test value, which is similar to the conclusion of Wang et al. ${ }^{[41]}$ when analyzing $e$ value of maize kernels in different shapes. In addition, as $H_{a}$ increased, $e_{s}$ decreased since bigger $H_{a}$ increased the rice straw-steel plate collision deformation. Due to extremely short collision contact time, rice straw consumes more energy in deformation and has decreased rebound speed after the collision. Therefore, $e_{s}$ decreased. This is similar to the reason for smaller $e$ value under greater drop height when Yang et al. ${ }^{[32]}$ calibrates $e$ value of castor capsule and Q235 steel.

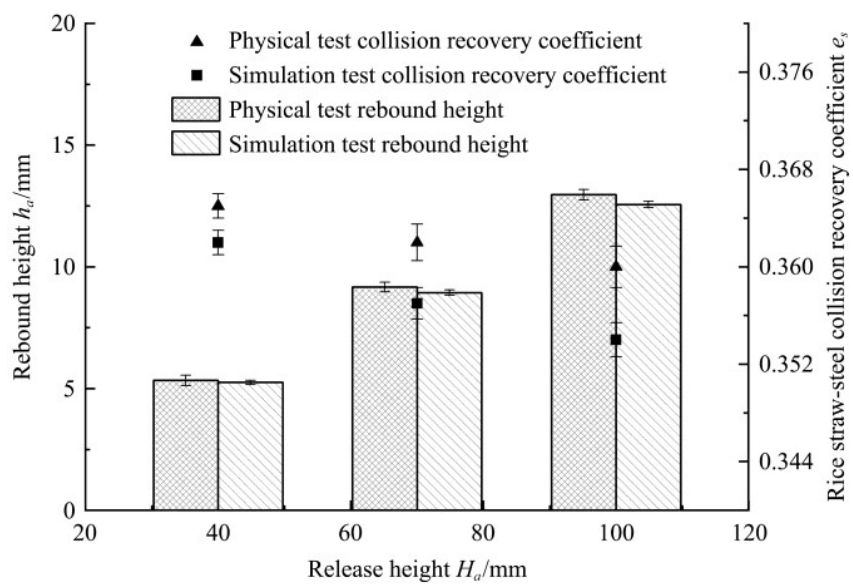

Figure 9 Impact of different release heights on collision recovery coefficient

\subsubsection{Rice straw-steel static friction coefficient}

Three sets of inclined plate physical tests are performed with each set repeated 5 times to take the average value. The resulting 
$\alpha_{1}$ is $20.03^{\circ}, 21.11^{\circ}$ and $19.25^{\circ}$, with an average value of $20.13^{\circ}$. The measured $\mu_{1}$ value was calculated as 0.367 . $\mu_{1}$ measured in the trial test had a value range of $0.152-0.536$, so $\mu_{1}$ level value in the simulation test can be derived therefrom. The simulation results are listed in Table 3. With the rice straw-steel static friction coefficient as the independent variable $x_{2}$ and the inclination angle as the dependent variable $y_{\alpha 1}$, the curve fitting equation for the test results is

$$
y_{\alpha 1}=-8.21 x_{2}^{2}+57.32 x_{2}+0.41
$$

Table 3 Simulation test results of rice straw-steel static friction

\begin{tabular}{ccc}
\hline Group number & Static friction coefficient $x_{2}$ & Inclination angle $y_{\alpha 1} /\left({ }^{\circ}\right)$ \\
\hline 1 & 0.15 & $9.62 \pm 0.05$ \\
2 & 0.25 & $14.66 \pm 0.07$ \\
3 & 0.35 & $20.39 \pm 0.08$ \\
4 & 0.45 & $25.21 \pm 0.10$ \\
5 & 0.55 & $30.13 \pm 0.13$ \\
\hline
\end{tabular}

The determination coefficient $R^{2}=0.999 . \quad y_{\alpha 1}=20.13^{\circ}$, so $x_{2}=$ 0.363 . Set $\mu_{1}$ as 0.363 . According to the three sets of repeated simulation tests, $\alpha_{1}$ was $18.14^{\circ}, 17.62^{\circ}$, and $19.16^{\circ}$, respectively. Paired $t$-test with the physical test value showed an insignificant difference between the two $(p>0.05)$, so the $\mu_{1}$ calibration value was determined as 0.363 .

The error between the $\mu_{1}$ simulated value and the physical test value is due to the natural properties and uneven physical form of the rice straw in the physical test relative to the simulation model. This is similar to the study on the static friction coefficient of potatoes by Liu et al. ${ }^{[19]}$ The surface roughness and viscous damping effect of rice straw in the physical test resulted in a bigger physical test value than the simulated value, which is consistent with the analysis result of DEM simulation friction coefficient of the glass bead particles calibrated by Angus et al. ${ }^{[42]}$ in the shear-cell test.

\subsubsection{Rice straw-steel rolling friction coefficient}

Three sets of slope physical tests were performed, with each set repeated 5 times to take the average value. The obtained $s$ was $149.21 \mathrm{~mm}, 151.46 \mathrm{~mm}, 150.42 \mathrm{~mm}$, with an average value of $150.36 \mathrm{~mm}$. The calculated $\mu_{1}^{\prime}$ was $0.211 . \quad \mu_{1}^{\prime}$ level value in the simulation test can be determined based on $\mu_{1}^{\prime}$ range of 0.149-0.343 in the preliminary test. The simulation results are listed in Table 4. With rice straw-steel rolling friction coefficient as the independent variable $x_{3}$ and the rolling distance as the dependent variable $y_{s}$, the curve fitting equation for the test results is

$$
y_{s}=3880.50 x_{3}^{2}-2857.80 x_{3}+576.52
$$

Table 4 Simulation test results of rice straw-steel rolling friction

\begin{tabular}{ccc}
\hline Group number & Rolling friction coefficient $x_{3}$ & Rolling distance $y_{s} / \mathrm{mm}$ \\
\hline 1 & 0.150 & $240.87 \pm 1.25$ \\
2 & 0.175 & $190.44 \pm 1.37$ \\
3 & 0.200 & $157.05 \pm 1.58$ \\
4 & 0.225 & $126.83 \pm 1.62$ \\
5 & 0.250 & $106.09 \pm 1.89$ \\
6 & 0.275 & $86.35 \pm 2.04$ \\
7 & 0.300 & $73.03 \pm 2.13$ \\
8 & 0.325 & $58.53 \pm 2.24$ \\
\hline
\end{tabular}

The determination coefficient $R^{2}=0.996$. $y_{s}=150.36$, so $x_{3}=$ 0.208 . Set $\mu_{1}^{\prime}$ as 0.208 , and by three sets of repeated simulation tests, resulting $s$ is respectively $151.84 \mathrm{~mm}, 154.46 \mathrm{~mm}$ and
$152.50 \mathrm{~mm}$. Paired $t$-test with the physical test value showed an insignificant difference between the two $(p>0.05)$, so $\mu_{1}^{\prime}$ was calibrated as 0.208

The error between $\mu_{1}^{\prime}$ simulated value and physical test value is due to the uneven rice straw surface morphology, invisible dust to the naked eye in the physical test, which makes the physical test value greater than the simulated value. This is similar to the reason for the calibration error in DEM simulation friction coefficient of glass bead particles according to Angus et al ${ }^{[42]}$. Steel plate surface roughness and lubrication treatment mode also explain the error between simulated value and physical test value. Ketterhagen et al. ${ }^{[36]}$ also showed that the collision material surface state inevitably impacts the rolling friction coefficient. In addition, there was a minor difference between the rice straw shape and the simulation model in the physical test, which led to deviations of the rice straw rolling trajectory from the simulation. The rolling distance then becomes smaller, so $\mu_{1}^{\prime}$ the physical test value was greater than the simulation value. As confirmed by Nguyen et al. ${ }^{[43]}$, the difference between soybean shape and sphere affects the grain trajectory and therefore rolling friction coefficient.

\subsection{Analysis on calibration results of rice straw-rice straw contact parameters}

3.2.1 Rice straw-rice straw collision recovery coefficient

Three sets of rice straw-steel collision physical tests with $H_{a}$ at $40 \mathrm{~mm}, 70 \mathrm{~mm}$, and $100 \mathrm{~mm}$ are performed, with each test repeated 15 times to get average $H_{b}$ values of $9.45 \mathrm{~mm}, 10.36 \mathrm{~mm}, 11.52 \mathrm{~mm}$, and average $H_{c}$ values of $21.15 \mathrm{~mm}, 27.14 \mathrm{~mm}, 33.12 \mathrm{~mm}$. The measured $e_{r}$ values were $0.241,0.238,0.236$, respectively. Perform simulation test with $H_{0}=70 \mathrm{~mm}$ and corresponding verification values of $H_{b}$ and $H_{c}$. The measured $e_{r}$ had a range of 0.181-0.345. $e_{r}$ level value and simulation result are shown in Table 5. With rice straw- rice straw collision recovery coefficient as the independent variable $x_{4}$ and the first collision height $y_{b}$ and $y_{c}$ as the dependent variables, the curve fitting equation for the simulation test is derived as:

$$
\begin{aligned}
& y_{b}=11.28 x_{4}^{2}-28.73 x_{4}+17.35 \\
& y_{c}=10.14 x_{4}^{2}+42.35 x_{4}+17.88
\end{aligned}
$$

Table 5 Simulation test results of rice straw-rice straw collision

\begin{tabular}{cccc}
\hline $\begin{array}{c}\text { Group } \\
\text { number }\end{array}$ & $\begin{array}{c}\text { Collision recovery } \\
\text { coefficient } x_{4}\end{array}$ & $\begin{array}{c}\text { First collision height } \\
y_{b} / \mathrm{mm}\end{array}$ & $\begin{array}{c}\text { First collision height } \\
y_{c} / \mathrm{mm}\end{array}$ \\
\hline 1 & 0.181 & $12.52 \pm 0.92$ & $26.08 \pm 0.88$ \\
2 & 0.236 & $11.18 \pm 1.05$ & $27.81 \pm 1.16$ \\
3 & 0.291 & $9.96 \pm 1.18$ & $30.77 \pm 1.13$ \\
4 & 0.346 & $8.85 \pm 1.14$ & $35.54 \pm 0.96$ \\
5 & 0.450 & $6.39 \pm 0.92$ & $38.46 \pm 1.07$ \\
6 & 0.550 & $5.11 \pm 0.89$ & $42.59 \pm 1.12$ \\
7 & 0.650 & $3.79 \pm 1.22$ & $49.67 \pm 1.25$ \\
8 & 0.750 & $1.95 \pm 1.31$ & $56.82 \pm 1.43$ \\
9 & 0.850 & $0.91 \pm 1.41$ & $61.16 \pm 1.54$ \\
10 & 1.000 & 0 & 70.00 \\
\hline
\end{tabular}

The determination coefficients were 0.998 and 0.996 . By substituting $y_{b}=0.36, y_{c}=27.14$ into the equation, $x_{4}=0.230$. Set $e_{r}$ to 0.230 . Three sets of repeated simulation tests were performed with $H_{0}$ at $40 \mathrm{~mm}, 70 \mathrm{~mm}, 100 \mathrm{~mm}$ to get average $H_{b}$ values of $9.57 \mathrm{~mm}, 10.78 \mathrm{~mm}, 11.64 \mathrm{~mm}$, with average $H_{c}$ values of $20.87 \mathrm{~mm}$, $27.22 \mathrm{~mm}, 32.37 \mathrm{~mm}$. Paired $t$-test with physical test values indicated no significant difference between the simulation and measured results $(p>0.05)$, so $e_{r}$ was calibrated as 0.230 . 
The reason for the difference between $e_{r}$ simulated value and The physical test value is similar to the finding of Wang ${ }^{[33]}$ when measuring $e_{r}$ of two maize kernels. It is because the two rice straws have stable collisions in the simulation, while random error in the physical test will cause slight deflection in the rice straw collision. Rice straw in the physical test has natural attributes different from the rice straw model, resulting in errors between the two. The test found that as $H_{0}$ increased, $e_{r}$ decreased. The reason was that bigger $H_{0}$ increased the potential energy of the released rice straw, the deformation increased while decreasing $e_{r}$. This is consistent with the conclusion of Yang et al. ${ }^{[32]}$ when calibrating the collision recovery coefficient between castor capsules.

3.2.2 Rice straw-rice straw static friction coefficient and rolling friction coefficient

The rice straw angle of repose was determined by three sets of physical tests by the split cylinder method. Each test was repeated 5 times to obtain the measured angle of repose of $34.17^{\circ}, 33.35^{\circ}$ and $32.92^{\circ}$, and the average value of $33.48^{\circ}$.

After the pre-simulation test, it was determined that $\mu_{2}$ and $\mu_{2}^{\prime}$ have value ranges of $0.35-0.45$ and $0.06-0.08$, respectively. Central Composite Design (CCD) test was performed by Design-Expert software. The test factors were the static friction coefficient $A$ and rolling friction coefficient $B$ between the rice straw. With test index as simulation angle of repose $Y$, the test factors are coded as shown in Table 6. The angle of repose is simulated with results shown in Table 7. The second-order regression model fitted by the simulation results is

$$
Y=70.45-119.13 A-475.13 B+150 A B+148.6 A^{2}+3115 B^{2}
$$

Table 6 Coding of simulation test factors

\begin{tabular}{ccc}
\hline \multirow{2}{*}{ Coding } & \multicolumn{2}{c}{ Factor } \\
\cline { 2 - 3 } & $\begin{array}{c}\text { Static friction coefficient } \\
\text { between rice straws } A\end{array}$ & $\begin{array}{c}\text { Rolling friction coefficient } \\
\text { between rice straws } B\end{array}$ \\
\hline 1.414 & 0.470 & 0.084 \\
1 & 0.450 & 0.080 \\
0 & 0.400 & 0.070 \\
-1 & 0.350 & 0.060 \\
-1.414 & 0.329 & 0.056 \\
\hline
\end{tabular}

Table 7 Simulation test results of angle of repose

\begin{tabular}{cccc}
\hline \multirow{2}{*}{$\begin{array}{c}\text { Serial } \\
\text { number }\end{array}$} & \multicolumn{2}{c}{ Test factor } & Evaluation index \\
\cline { 2 - 4 } \cline { 3 - 4 } & $\begin{array}{c}\text { Static friction } \\
\text { coefficient } A\end{array}$ & $\begin{array}{c}\text { Rolling friction } \\
\text { coefficient } B\end{array}$ & $\begin{array}{c}\text { Simulated angle of } \\
\text { repose } Y /\left(^{\circ}\right)\end{array}$ \\
\hline 1 & -1 & -1 & 32.85 \\
2 & 1 & -1 & 33.52 \\
3 & -1 & 1 & 33.27 \\
4 & 1 & 1 & 34.24 \\
5 & -1.414 & 0 & 32.65 \\
6 & 1.414 & 0 & 34.39 \\
7 & 0 & -1.414 & 33.21 \\
8 & 0 & 1.414 & 33.59 \\
9 & 0 & 0 & 32.88 \\
10 & 0 & 0 & 33.01 \\
11 & 0 & 0 & 32.52 \\
12 & 0 & 0 & 32.86 \\
13 & 0 & 0 & 32.64 \\
\hline
\end{tabular}

The model's significance test has $p<0.001$, determination coefficient $R^{2}=0.932$, correction absolute coefficient $R_{\text {adj }}^{2}=0.884$, which are all close to 1 , indicating that the regression model can well predict the target angle of repose. With lack of fit $p=0.4444>$ 0.05 , coefficient of variation $C V=0.61 \%$, the model fits well. The regression variance analysis of the test results is shown in Table 8. The analysis shows that static friction coefficient $A$, quadratic term $A^{2}$ and rolling friction coefficient $B$ have a significant effect on the angle of repose $(p<0.01)$; quadratic term $B^{2}$ of rolling friction coefficient has a significant effect on the angle of repose $(p<0.05)$. Ma et al. ${ }^{[20]}$ confirmed that the static friction coefficient between alfalfa straws, its quadratic term and the rolling friction coefficient had a significant effect on the angle of repose, while the quadratic term of the rolling friction coefficient had a significant effect on the angle of repose. The software optimization module was used for optimization so that the simulated angle of repose was closest to the physical test value of $33.48^{\circ}$. Thus, $\mu_{2}$ and $\mu_{2}^{\prime}$ could be obtained as 0.44 and 0.07 .

Table 8 Analysis of variance of CCD test results

\begin{tabular}{ccccc} 
Source of variation & Sum of square & Degree of freedom & Mean square & $p$-value \\
\hline Model & 3.930 & 5 & 0.790 & $0.0006^{* *}$ \\
$A-A$ & 2.100 & 1 & 2.100 & $0.0002^{* *}$ \\
$B-B$ & 0.350 & 1 & 0.350 & $0.0048^{* *}$ \\
AB & 0.022 & 1 & 0.022 & 0.4824 \\
$A^{2}$ & 0.960 & 1 & 0.960 & $0.0019^{* *}$ \\
$B^{2}$ & 0.680 & 1 & 0.680 & $0.0219^{*}$ \\
Residual & 0.290 & 7 & 0.041 & \\
Lack of fit & 0.130 & 3 & 0.043 & 0.4444 \\
Pure error & 0.160 & 4 & 0.039 & \\
Total dispersion & 4.210 & 12 & & \\
\hline Note: ** shows the significance at $p<0.01 ; *$ shows the significance at $p<0.05$
\end{tabular}

\subsection{Analysis of contact parameter verification results}

Three sets of simulation tests were performed by the split cylinder method based on contact parameter calibration values in Table 9. Each test was repeated 5 times to get average simulated angles of repose of $33.76^{\circ}, 34.04^{\circ}, 32.43^{\circ}$. Paired $t$-test with the physical test values showed an insignificant difference between the measured and simulated values $(p>0.05)$.

Table 9 Discrete element simulation contact parameters

\begin{tabular}{ll}
\hline \multicolumn{1}{c}{ Parameter } & Value \\
\hline Rice straw-steel collision recovery coefficient & 0.357 \\
Rice straw-steel static friction coefficient & 0.363 \\
Rice straw-steel rolling friction coefficient & 0.208 \\
Rice straw-rice straw collision recovery coefficient & 0.230 \\
Rice straw-rice straw static friction coefficient & 0.440 \\
Rice straw-rice straw rolling friction coefficient & 0.070 \\
\hline
\end{tabular}

Using the side plate lifting method, Zhang et al. ${ }^{[17]}$ measured radial accumulation angle of maize straw of different lengths, revealing insignificant differences between the simulation results and the physical test value. Wen et al. ${ }^{[24]}$ determined that the angle of repose of urea particles with different moisture contents in the side-plate lifting test, proving the insignificant difference between the simulation and the physical test value, so it was concluded that the side-plate lifting method is a universally applicable method in the calibration of discrete element contact parameter.

Taking into account different rice straw lengths after harvest, this study adopted a side-plate lifting method to determine the angle of repose of 10-100 mm rice straw, as shown in Figure 10. As shown in Table 10, the paired $t$-test between the simulation test results and the physical test values showed an insignificant 
difference between the two $(p>0.05)$. Also using steel side plate, Wen et al. ${ }^{[31]}$ measured the angle of repose of sugarcane stalks in different lengths, confirming that the simulated value was not significantly different from the real experimental value. It indicated that the contact parameters calibrated herein have universal applicability to the discrete element simulation study of rice straw-rice straw and rice straw-agricultural machinery parts.

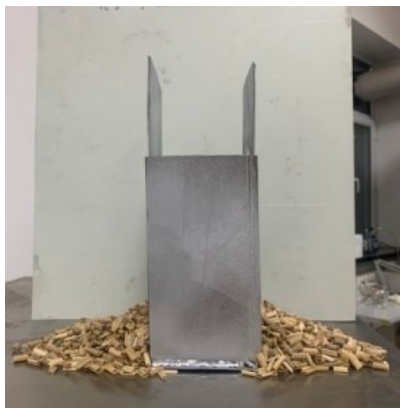

a. Physical test

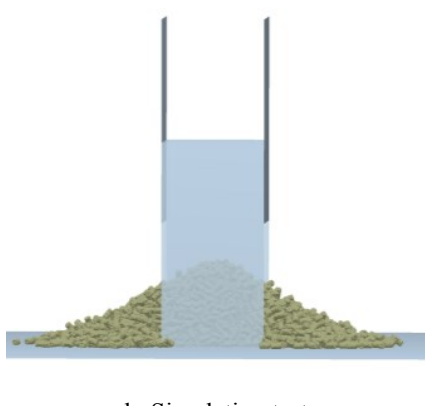

b. Simulation test

Figure 10 Angle of repose test by side plate lifting method

Table 10 Angles of repose of rice straw in different lengths

\begin{tabular}{lccccc}
\hline \multirow{2}{*}{ Parameter } & \multicolumn{5}{c}{ Rice straw length $/ \mathrm{mm}$} \\
\cline { 2 - 6 } & 10 & 30 & 50 & 70 & 100 \\
\hline Test angle of repose $/\left(^{\circ}\right)$ & 33.43 & 34.31 & 36.06 & 37.42 & 40.17 \\
Simulation angle of repose $/\left(^{\circ}\right)$ & 32.85 & 33.23 & 34.13 & 35.06 & 36.65 \\
\hline
\end{tabular}

\section{Conclusions}

Based on Hertz-Mindlin (no slip) model of DEM, this study combined physical test and simulation test to calibrate rice straw-steel collision recovery coefficient, static friction coefficient and rolling friction coefficient as $0.357,0.363$ and 0.208 , respectively. Rice straw-rice straw collision recovery coefficient, static friction coefficient and rolling friction coefficient were calibrated as $0.230,0.44$ and 0.07 , respectively. There was an insignificant difference between the verification simulation value and physical test value under this calibration value $(p>0.05)$. Also, no significant difference was found between the simulated and physical test value of rice straw angle of repose under different lengths $(p>0.05)$. This study provides key contact parameters for the discrete element simulation of rice straw and its related machinery, which help with the design and optimization improvement of agricultural machinery and tools, and promotes the development process in the field of agricultural machinery.

\section{Acknowledgements}

The authors acknowledge that this work was financially supported by the National Natural Science Foundation of China (Grant No. 31901408); the Natural Science Foundation of Heilongjiang Province (Grant No. LH2020E097); the Science and Technology Development Project of Jilin Province (Grant No. 20200402100NC; 20200201206JC); 2020 China Russia Young Scientists Communication Project.

\section{[References]}

[1] Tunio M H, Gao J M, Talpur M A, Lakhiar I A, Chandio F A, Shaikh S A, et al. Effects of different irrigation frequencies and incorporation of rice straw on yield and water productivity of wheat crop. Int J Agric \& Biol Eng, 2020; 13(1): 138-145.

[2] Li L Q, Wang D F, Yang X. Study on round rice straw bale wrapping silage technology and facilities. Int J Agric \& Biol Eng, 2018; 11(4):
$88-95$.

[3] Jia H L, Huang D Y, Qi J T, Zhuang J, Yuan H F, Zhao J L, et al. Conservation tillage in northern dry crop areas. In: Modern agricultural machinery design theory and application. Beijing: Science Press, 2020; pp.36-38. (in Chinese)

[4] He Jin, Li H W, Chen H T, Lu C Y, Wang Q J. Research progress of conservation tillage technology and machine. Transactions of the CSAM, 2018; 49(4): 1-19. (in Chinese)

[5] Zhang T, Xia J F, Wu G, Zhai J B. Automatic navigation path detection method for tillage machines working on high crop stubble fields based on machine vision. Int J Agric \& Biol Eng, 2014; 7(4): 29-37.

[6] Wang D F, Buckmaster D R, Jiang Y Y. Experimental study on baling rice straw silage. Int J Agric \& Biol Eng, 2017; 4(1): 20-25.

[7] Park S I, Yang H I, Park H J, Seo B S, Jeong Y J, Lim S S, et al. Rice straw cover decreases soil erosion and sediment-bound $\mathrm{C}, \mathrm{N}$, and P losses but increases dissolved organic $\mathrm{C}$ export from upland maize fields as evidenced by $\delta^{13} \mathrm{C}$. Science of the Total Environment, 2020; 753: 1-11.

[8] Shi L R, Zhao W Y, Sun B G, Sun W. Determination of the coefficient of rolling friction of irregularly shaped maize particles by using discrete element method. Int J Agric \& Biol Eng, 2020; 13(2): 15-25.

[9] Dai F, Song X F, Guo W J, Zhao W Y, Zhang F W, Zhang S L. Simulation and test on separating cleaning process of flax threshing material based on gas-solid coupling theory. Int J Agric \& Biol Eng, 2020; 13(1): 73-81.

[10] Zhang R, Han D L, Ji Q L, He Y, Li J Q. Calibration methods of sandy soil parameters in simulation of discrete element method. Transactions of the CSAM, 2017; 48(3): 49-56. (in Chinese)

[11] Ucgul M, Fielke J.M, Saunders C. 3D DEM tillage simulation: Validation of a hysteretic spring (plastic) contact model for a sweep tool operating in a cohesionless soil. Soil and Tillage Research, 2014; 144(1): 220-227.

[12] Fang H M. Research on the straw-soil-rotary blade interaction using discrete element method. Doctoral dissertation. Nanjing: Nanjing Agricultural University, 2016; pp.53-83. (in Chinese)

[13] Lenaerts B, Aertsen T, Tijskens E, Ketelaere B D, Ramon H, De Baerdemaeker $\mathrm{J}$, et al. Simulation of grain-straw separation by discrete element modeling with bendable straw particles. Computers and Electronics in Agriculture, 2014; 101: 24-33.

[14] Zhang X M. Study on working mechanism and experimental of rotary tiller for high straw returning. Doctoral dissertation. Wuhan: Huazhong Agricultural University, 2017; pp.92-106. (in Chinese)

[15] Liu F Y, Zhang J, Chen J. Modeling of flexible wheat straw by discrete element method and its parameters calibration. Int J Agric \& Biol Eng, 2018; 11(3): 42-46.

[16] Zhou H, Li D, Liu Z Y, Li Z Y, Luo S C, Xia J F. Simulation and experiment of spatial distribution effect after straw incorporation into soil by rotary burial. Transactions of the CSAM, 2019; 50(9): 69-77. (in Chinese)

[17] Zhang T, Liu F, Zhao M Q, Ma Q, Wang W, Fan Q, et al. Determination of corn stalk contact parameters and calibration of Discrete Element Method simulation. Journal of China Agricultural University, 2018; 23(4) 120-127. (in Chinese)

[18] Wu S, Li P P, Zhang X L, Xu Y F, Liu J Z, Wang J Z. Vertical spiral mixing mechanism of tomato straw particles based on discrete element method. Journal of Drainage and Irrigation Machinery Engineering, 2018 36(8): 719-724. (in Chinese)

[19] Liu W Z, He J, Li H W, Li X Q, Zheng K, Wei Z C. Calibration of simulation parameters for potato minituber based on EDEM. Transactions of the CSAM, 2018; 49(5): 125-135, 142. (in Chinese)

[20] González-Montellano C, Fuentes J M, Ayuga-Téllez E, Ayuga F. Determination of the mechanical properties of maize grains and olives required for use in DEM simulations. Journal of Food Engineering, 2012; 111(4): 553-562.

[21] Agricultural Mechanization Management Division. Mechanized returning technology mode of main crop straw. Available: http://www.njhs.moa.gov.cn/keji/201712/t20171227_6129152.htm. Accessed on [2018-09-12]. (in Chinese)

[22] Luo Y J, Chen M H, Mu L, Hu L X, Zhang Z F, Gao S, et al. Effects on silage quality of mixing different ratios of rice straw with alfalfa and wheat bran. Acta Prataculturae Sinica, 2019; 28(5): 178-184. (in Chinese)

[23] Ma Y H, Song C D, Xuan C Z, Wang H Y, Yang S, Wu P. Parameters calibration of discrete element model for alfalfa straw compression simulation. Transactions of the CSAE, 2020; 36(11): 22-30. (in Chinese) 
[24] Wen X Y, Yuan H F, Wang G, Jia H L. Calibration method of friction coefficient of granular fertilizer by discrete element simulation. Transactions of the CSAM, 2020; 51(2):116-122, 142. (in Chinese)

[25] Cao Z X. Study on preparation and wear properties of wear-resistant coatings containing diamond for agricultural machinery. Master dissertation. Guangzhou: Zhongkai University of Agriculture Engineering, 2019; pp.10-16. (in Chinese)

[26] Huo L L, Tian Y S, Zhao L X, Yao Z L, Hou S L, Meng H B. Research on physical property of crop straw and test methods. Renewable Energy Resources, 2011; 29(6): 86-92. (in Chinese)

[27] Fang H M, Ji C Y, Tagar A A, Zhang Q Y, Guo J. Simulation analysis of straw movement in straw-soil-rotary blade system. Transactions of the CSAM, 2016; 47(1): 60-67. (in Chinese)

[28] Ucgul M, Fielke J M, Saunders C. Three-dimensional discrete element modelling of tillage: Determination of a suitable contact model and parameters for a cohesionless soil. Biosystems Engineering, 2014; 121: $105-117$.

[29] Lu Y G, Wu N, Wang B, Yu Z Y, Lin D Z, Hu Z C. Measurement and analysis of peanuts' restitution coefficient in point-to-plate collision mode. Journal of China Agricultural University, 2016; 21(8): 111-118. (in Chinese)

[30] Sharma R K, Bilanski W K. Coefficient of restitution of grains. Transactions of the ASAE, 1971; 14: 216-218.

[31] Wen X, Yang W, Guo W J, Zeng B S. Parameter determination and validation of discrete element model of segmented sugarcane harvester for impurity removal. Journal of Chinese Agricultural Mechanization, 2020; 41(1): 12-18. (in Chinese)

[32] Yang Y, Hou J M, Bai J B, Yao E C, He T, Li J P. Determination and analysis of typical castor seed collision restitution coefficient. Journal of Chinese Agricultural University, 2019; 24(1): 138-148. (in Chinese)

[33] Wang Y. Simulation analysis and experimental study of the screening process of a swing-bar sieve based on the coupling of DEM with MBK. Doctoral dissertation. Changchun: Jilin University, 2018; pp.44-49. (in
Chinese)

[34] Shi L R, Sun W, Zhao W Y, Yang X P, Feng B. Parameter determination and validation of discrete element model of seed potato mechanical seeding Transactions of the CSAE, 2018; 34(6): 35-42. (in Chinese)

[35] American Society for Testing and Materials. Standard test method for measuring rolling friction characteristics of a spherical shape on a flat horizontal plane: G 194-08. West Conshohocken, PA: American Society for Testing and Materials, 2009; pp.17-21.

[36] Ketterhagen W R, Bharadwaj R, Hancock B C. The coefficient of rolling resistance (CoRR) of some pharmaceutical tablets. International Journal of Pharmaceutics, 2010; 392: 107-110.

[37] Wong C X, Daniel M C, Rongong J A. Energy dissipation prediction of paticle dampers. Journal of Sound and Vibration, 2009; 319(1-2): 91-118.

[38] Sun H N, Chen X, Xia C Y. Measurement and experiment study on angle of repose of paddy. Modern Food, 2018; 2: 63-66. (in Chinese)

[39] Liao Y T, Liao Q X, Zhou Y, Wang Z T, Jiang Y J, Liang F. Parameters calibration of discrete element model of fodder rape crop harvest in bolting stage. Transactions of the CSAM, 2020; 51(6): 73-82. (in Chinese)

[40] Liu F Y, Zhang J, Li B, Chen J. Calibration of parameters of wheat required in discrete element method simulation based on repose angle of particle heap. Transactions of the CSAE, 2016; 32(12): 247-253. (in Chinese)

[41] Wang L J, Zhou W X, Ding Z J, Li X X, Zhang C E. Experimental determination of parameter effects on the coefficient of restitution of differently shaped maize in three-dimensions. Powder Technology, 2015; 284: 187-194.

[42] Angus A, Yahla L A A, Malone R, Khala M, Hare C, Ozel A, et al Calibrating friction coefficients in discrete element method simulations with shear-cell experiments. Powder Technology, 2020; 372: 290-304.

[43] Nguyen T X, Le L M, Nguyen T C, Nguyen N T H, Le T T, Pham B T, et al. Characterization of soybeans and calibration of their DEM input parameters. Particulate Science and Technology, 2020; 39(6): 1-19. 